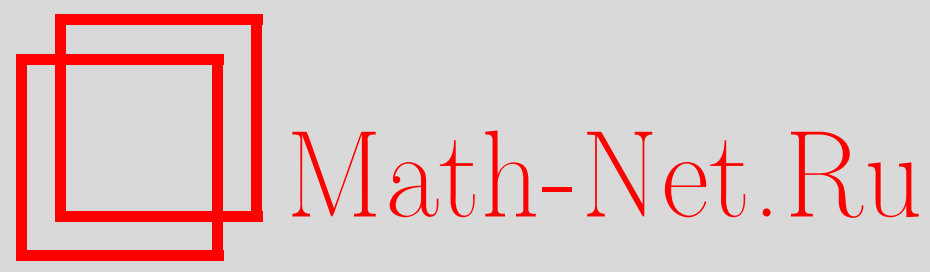

K. В. Рерих, Общий подход к интегрированию обратимых динамических систем, определяемых отображениями из группы Кремоны бирациональных преобразований $\operatorname{Cr}\left(P_{k}^{n}\right)$, Матем. заметки, 2000, том 68, выпуск 5, 699-709

DOI: https://doi.org/10.4213/mzm991

Использование Общероссийского математического портала Math-Net.Ru подразумевает, что вы прочитали и согласны с пользовательским соглашением http://www.mathnet.ru/rus/agreement

Параметры загрузки:

IP: 44.207 .124 .84

26 апреля 2023 г., $17: 59: 01$

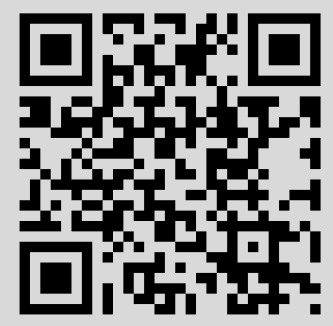




\title{
ОБЩИЙ ПОДХОД К ИНТЕГРИРОВАНИЮ \\ ОБРАТИМЫХ ДИНАМИЧЕСКИХ СИСТЕМ, ОПРЕДЕЛЯЕМЫХ ОТОБРАЖЕНИЯМИ ИЗ ГРУППЫ КРЕМОНЫ БИРАЦИОНАЛЬНЫХ ПРЕОБРАЗОВАНИЙ $\operatorname{Cr}\left(P_{k}^{n}\right)$
}

К. В. Рерих

\begin{abstract}
Развит общий подход к интегрированию обратимых динамических систем, определяемых композицией двух инволюций: нелинейной - стандартного преобразования Кремоны, и линейной. Благодаря теореме М. Нётера интегрирование таких систем является основой для интегрирования шшрокого класса кремоновых динамических систем. В работе получены функциональное уравнение для инвариантных однородных полиномов и достаточные условия для алгебраической интегрируемости рассматриваемых систем. Установлена применимость теоремы Зигеля о линеаризации отображения в случае, когда собственные числа отображения в неподвижной точке являются алгебраическими числами.
\end{abstract}

Библиография: 42 названия.

1. Введение. Обратимые динамические системы (ОДС) занимают важное место в физике, что связано с инвариантностью многих физических законов относительно обращения времени. Обратимые динамические системы, как известно (см. [1]-[6]), качественно подобны гамильтоновым системам. В частности, доказано существование тора Колмогорова-Арнольда-Мозера в обратимых негамильтоновых потоках [1], [2] и отображениях с неподвижной эллиптической точкой [4]-[6]. Напомним определение обратимого отображения.

ОПРЕДЕЛЕНИЕ. Отображение $\Phi$ назьвается обратимым, если имеется симметрия $G$ такая, что $\Phi^{-1}=G \circ \Phi \circ G$ и $G$ есть инволюция: $G^{2}=\operatorname{id~[7].~Из~этого~определения~}$ следует, что $\Phi \circ G$ есть также инволюция, а $\Phi$ - композиция двух инволюций.

В известных примерах интегрируемых обратимых отображений (см. [8]) последние являются частными случаями плоских отображений (преобразований) Кремоны (см. [9], $\left[10\right.$, т. 3, с. 94]) - бирациональных преобразований в проективном пространстве $P_{k}^{n}$ (где $k$-поле комплексных $(\mathbb{C})$ или действительных $(\mathbb{R})$ чисел). Известно (теорема М. Нётера [9], [10]), что при $n=2$ группа $\operatorname{Cr}\left(P_{k}^{n}\right)$ разрешима посредством стандартных (квадратичных) преобразований Кремоны. Хотя при $n \geqslant 3$ ситуация сложнее, по-видимому основой для интегрирования произвольных кремоновых ОДС может служить исследование интегрируемости ОДС, определяемьг композицией стандартного отображения 
Кремоны $I \in \operatorname{Cr}\left(P_{k}^{n}\right)$, являющегося инволюцией $\left(I^{2}=\mathrm{id}\right)$, и линейного отображения, задаваемого инволютивной матрицей $A$.

Динамические системы такого вида возникли в статической модели дисперсионного подхода $[11, \S 29]$. Недавно в [12] был получен неалгебраический первьй интеграл для одной системы этого типа. Укажем также, что проблема интегрирования динамических систем, определяемых кремоновьми отображениями, исследована для афинной подгрупшы Кремона GA, (полиномиальных отображений) в [13] и [14].

Итак, после этого введения и сделанных замечаний мы остановимся в п. 2 на формулировкепроблемы и истории вопроса. В п. 3 будет получено функциональное уравнение на инвариантные однородные полиномы, решения которого мы будем назьвать парциальньми автоморфньми формами. В п. 4 на этой основе будут установлены достаточные условия алгебраической интегрируемости таких динамических систем, обсуждается вопрос об интегрировании таких систем с позиций локальной теории диффеоморфизмов, дается формулировка и доказательство теоремы, дополнительной к теореме Зигеля (см. [15, гл. $6, \S 2],[16, \S \S 25,28])$ и следующей из теорем А. Бэйкера, Н. И. Фельдмана [17]-[20] для линейных форм от логарифмов алгебраических чисел.

2. Формулировка проблемы. Рассматриваются обратимые динамические системы вида

$$
\begin{gathered}
S(-w)=A S(w), \\
S(w+1)=I \circ A S(w),
\end{gathered}
$$

где $A$ - линейная, а $I$ - нелинейная инволюции, т.е. $A^{2}=\mathrm{id}, I^{2}=\mathrm{id}$ :

a) $S(w)=\left(S_{1}(w), S_{2}(w), \ldots, S_{n}(w)\right), w \in \mathbb{C}, S(w) \in M^{n}(\mathbb{C}), M(\mathbb{C})$ - функциональное пространство мероморфных функций, $S^{*}(w)=S\left(w^{*}\right)$, где “*” - операция комплексного сопряжения;

б) числовая матрица кроссинг-симметрии $A$, определяемая группой симметрии статического гамильтониана и квантовыми числами (орбитальным моментом $L$, спиновым - $J$, изоспиновым - $T$ ) сталкивающихся частиц, обладает некоторыми общими свойствами:

$$
A^{2}=\mathrm{id}, \quad A_{i j} \in \mathbb{Q} \text { или } \mathbb{R}, \quad i, j \in(1,2, \ldots, n),
$$

а так как $\operatorname{det} A \neq 0$, то $A$ - матрища простой структуры и

$$
\begin{gathered}
A=B \Lambda^{a} B^{-1}, \quad \Lambda^{a}=\operatorname{diag}\left(\lambda_{1}^{a}, \lambda_{2}^{a}, \ldots, \lambda_{n}^{a}\right), \quad \lambda_{j}^{a}= \pm 1, \\
B_{i j}=\mu_{i}^{(j)}, \quad A \mu^{(j)}=\lambda_{j}^{a} \mu^{(j)}, \quad i, j \in(1,2, \ldots, n),
\end{gathered}
$$

где $B$ - фундаментальная матрица в базисе собственных векторов $\mu^{(j)}$ матрицы $A[21$, с. 85] (конкретные реализации и другие свойства см. [22], [23]);

в) $A \mu^{(n)}=\mu^{(n)}, \mu^{(n)}=(1,1, \ldots, 1)$;

г) $I^{2}=\mathrm{id},(I S)_{i}=1 / S_{i}, I \in \operatorname{Cr}\left(P_{k}^{n}\right)$, где $\operatorname{Cr}\left(P_{k}^{n}\right)$ - группа бирациональных преобразований Кремоны в проективном пространстве $P_{k}^{n}, k \equiv \mathbb{R}$ или $\mathbb{C}$, а $I$ - стандартное преобразование Кремоны (его обобщение на $n$-мерньй случай). 
ЗАмечаниЕ 1. Динамическая система (1), (2) обладает следующей группой автоморфизмов:

$$
S(w) \rightarrow S(w+\beta(w)) \exp \left(\alpha\left(w-\frac{1}{2}\right)\right)
$$

где $\beta(w) \in M(\mathbb{C})$ - для рациональных $S(w)$ или $\beta(w) \in E(\mathbb{C})(E(\mathbb{C})$ - функциональное пространство целых функций) - для трансцендентных мероморфных функций $S(w)$ и $\exp (\alpha(w)) \in M(\mathbb{C})$

$$
\begin{gathered}
\beta(w+1)=\beta(w), \quad \beta(-w)=-\beta(w), \\
\alpha(w+1)=-\alpha(w), \quad \alpha(-w)=-\alpha(w) .
\end{gathered}
$$

Очевидно, что группа автоморфизмов пространства решений динамической системы (1), (2) не исчерпьвается $(5),(6)$; более того, она должна включать в себя автоморфизмы, зависящие еще от $n-2$ произвольных функций $w$ периода 1.

ЗАмЕчание 2 . Пусть $\Phi$ - отображение $\left(\Phi \in \operatorname{Cr}\left(P_{k}^{n}\right)\right)$ и $Y(w)=\Phi S(w)$. Тогда $Y(w)$ есть решение уравнений

$$
\begin{aligned}
Y(-w) & =\left(\Phi \circ U^{-1} \circ \Lambda^{a} \circ\left(\Phi \circ U^{-1}\right)^{-1}\right) Y(w), \\
Y(w+1) & =\left(\Phi \circ I \circ U^{-1} \circ \Lambda^{a} \circ U \circ \Phi^{-1}\right) Y(w),
\end{aligned}
$$

где $A=U^{-1} \circ \Lambda^{a}$ 。 $U, \operatorname{Det} U \neq 0, U_{i j} \in \mathbb{R}(i, j=1,2, \ldots, n)$.

РЕзЮме. Динамическая система $(1),(2)$ является базовой для весьма широкого класса динамических систем.

Проблема нахождения явного вида всех $n-2$ первых интегралов динамической системы (1), (2) стоит уже почти 30 лет, начиная с [24], [25], где она была решена для $n=2$, частично в [22], [26], [27] для $n=3$ и в [22] для случая $n=4$, где были найдены частные решения динамической системы $(1),(2)$ в классе рациональных функций (без учета $(5),(6))[22],[26]$, или в классе мероморфных трансцендентных функций [27]. В [28] был предложен метод локального построения инвариантных многообразий в фазовом пространстве. Необходимость вьполнения некоторых локальных условий (при $w=0$ ) была мотивом поиска решения этой общей проблемы при $n=3$ и $n=4$ (см. [29], [30]).

В работе [29] эта проблема рассматривалась с позиций качественной теории дифференциальных уравнений: исследовалось дифференциальное уравнение абелевой группы итераций динамической системы (1), (2) в окрестности параболической неподвижной точки, что дало возможность получить [29] формальные расходящиеся общие решения. В [30], [31] был предложен другой подход к решению этой проблемы на основе использования общей теории преобразований Кремоны [9]. И наконец, в недавней работе автора [12] общая проблема решена для $n=3$ и матрицы $A=A(1,1)$, соответствующей процессу рассеяния двух частиц со спином 1.

3. Функциональное уравнение для инвариантных однородных полиномов. Введем в дополнение к $(3),(4) z(w), j(z)$ и $\pi(z): z(w) \in \mathbb{C}^{n}, j(z) \in \mathbb{C}^{n}, \pi(z) \in \mathbb{C}$,

$$
\begin{aligned}
z(w) & =B^{-1} S(w), \\
j(z) & =A B z \\
\pi(z) & =\prod_{i=1}^{n} j_{i}(z) .
\end{aligned}
$$


Тогда динамическая система (1), (2) примет вид

$$
\begin{gathered}
z(-w)=\Lambda^{a} z(w), \\
z(w+1)=\frac{\varphi(z(w))}{\pi(z(w))}
\end{gathered}
$$

где

$$
\varphi(z)=\pi(z) B^{-1}(I j(z)), \quad \Lambda^{a}=B^{-1} A B, \quad S(w)=j\left(\Lambda^{a} z(w)\right) .
$$

Пусть $m=\left(m_{1}, m_{2}, \ldots, m_{n}\right)$ - мультииндекс, $m_{i} \in \mathbb{Z}_{+},\left(\mathbb{Z}_{+}-\right.$множество цельх неотрицательных чисел), $|m|=\sum_{i=1}^{n} m_{i}$, и пусть $P_{k}(z)$ - однородный полином степени $k$. Тогда имеет место следующая теорема (мы не вьписываем зависимость от $w$ ).

ТЕОрема 1. Инвариантные неприводимые однородные полиномы $P_{k}(z)$ есть решения следующих функииональных уравнений:

$$
\begin{aligned}
P_{k}(\varphi(z)) & =\varepsilon P_{k}(z) j^{m}(z), \\
P_{k}\left(\Lambda^{a} z\right) & =\nu P_{k}(z),
\end{aligned}
$$

əде $j^{m}(z) \equiv j_{1}^{m_{1}} j_{2}^{m_{2}} \cdots j_{n}^{m_{n}},|m|=k(n-2) u \varepsilon, \nu= \pm 1$.

ДокАЗАТЕЛЬСТвО. Пусть $P_{k}(z)=0$ определяет некоторую инвариантную поверхность в $\mathbb{C}^{n}$ и $P_{k}(\mu z)=\mu^{k} P_{k}(z)$, где $\mu \in \mathbb{C}$. Тогда условие инвариантности этой поверхности есть $(\tilde{z}=z(w+1))$

$$
P_{k}(\tilde{z})=\frac{P_{k}(\varphi(z))}{\pi^{k}(z)}=P_{k}(z) \frac{\mathscr{M}_{N}(z)}{\pi^{k}(z)},
$$

где $N \equiv k(n-2), \mathscr{M}_{N}(z)$ - однородный полином степени $N$. Совершая в (12) замену $w \rightarrow-w-1$, получим

$$
P_{k}\left(\Lambda^{a} z\right)=P_{k}\left(\Lambda^{a} \tilde{z}\right) \frac{\mathscr{M}_{N}\left(\Lambda^{a} \tilde{z}\right)}{\pi^{k}\left(\Lambda^{a} \tilde{z}\right)} .
$$

Из инвариантности относительно замены $w \rightarrow-w$ следует

$$
P_{k}(z)=0 \Longrightarrow P_{k}\left(\Lambda^{a} \tilde{z}\right)=0 \Longrightarrow P_{k}\left(\Lambda^{a} z\right)=\nu P_{k}(z), \quad \nu= \pm 1
$$

Функции $j(z)$ преобразуются, как это следует из (10), (13) и (14):

$$
j^{\prime}(\tilde{z})=j\left(\Lambda^{a} \tilde{z}\right)=I j(z), \quad j^{\prime}(z) \stackrel{\text { def }}{=} j\left(\Lambda^{a} z\right) .
$$

Тогда из (18) следует

$$
P_{k}(z)=P_{k}(\tilde{z}) \mathscr{M}_{N}\left(\Lambda^{a} \tilde{z}\right) \pi^{k}(z) .
$$

Подставляя $(17)$ в $(21)$, получим $\mathscr{M}_{N}(z) \mathscr{M}_{N}\left(\Lambda^{a} \tilde{z}\right)=1$. Определим

$$
\mathscr{M}_{N}(z)=\mathscr{M}_{N}\left(B^{-1} A^{-1} j(z)\right) \stackrel{\text { def }}{=} \overline{\mathscr{M}}_{N}(j(z)) .
$$


Итак,

$$
\overline{\mathscr{M}}_{N}(j(z)) \overline{\mathscr{M}}_{N}\left(j^{\prime}(\tilde{z})\right)=1 .
$$

Так как $\overline{\mathscr{M}}_{N}(\mu j)=\mu^{N} \overline{\mathscr{M}}_{N}(j), N=k(n-2)$, то

$$
\overline{\mathscr{M}}_{N}(j)=\sum_{|m|=N} C_{N}^{m_{1} m_{2} \cdots m_{n}} j^{m},
$$

где $m \stackrel{\text { def }}{=}\left(m_{1}, m_{2}, \ldots, m_{n}\right), m_{i} \in \mathbb{Z}_{+}$, и $j^{m} \stackrel{\text { def }}{=} \prod_{i=1}^{n} j_{i}^{m_{i}}$.

Тогда имеем $\left(C_{N}^{m} \stackrel{\text { def }}{=} C_{N}^{m_{1} m_{2} \cdots m_{n}}\right)$

$$
\sum_{|m|=N} C_{N}^{m} j^{m} \sum_{\left|m^{\prime}\right|=N} C_{N}^{m^{\prime}} j^{\prime m^{\prime}}(\tilde{z})=1 .
$$

Из $(22)$ с учетом $(20)$ следует $\left(m-m^{\prime} \stackrel{\text { def }}{=} m^{\prime \prime} \in \mathbb{Z}^{n}\right)$

$$
\sum_{\left|m^{\prime \prime}\right|=0} j^{m^{\prime \prime}} \sum_{\substack{\left|m^{\prime}\right|=N \\\left|m^{\prime}+m^{\prime \prime}\right|=N}} C_{N}^{m^{\prime}+m^{\prime \prime}} C_{N}^{m^{\prime}}=1
$$

Из (23) имеем:

1) $m^{\prime \prime}=0, m_{i}^{\prime \prime}=0, i=1,2, \ldots, n$,

$$
\sum_{\left|m^{\prime}\right|=N}\left(C_{N}^{m^{\prime}}\right)^{2}=1
$$

2) $m^{\prime \prime} \neq 0,\left|m^{\prime \prime}\right|=0, m^{\prime \prime} \in \mathbb{Z}^{n},\left|m_{i}^{\prime \prime}\right| \leqslant N$,

$$
\sum_{\substack{\left|m^{\prime}\right|=N \\\left|m^{\prime}+m^{\prime \prime}\right|=N}} C_{N}^{m^{\prime}+m^{\prime \prime}} C_{N}^{m^{\prime}}=0 .
$$

Допустим, что при каком-то одном $m^{(1)}=\left(m_{1}^{(1)}, \ldots, m_{n}^{(1)}\right)\left|C_{N}^{m^{(1)}}\right|=1$. Тогда все остальные коэффициенты в силу (24) равны 0. Пусть теперь два коэффициента отличны от нуля: $C_{N}^{m^{(1)}}=\alpha$ и $C_{N}^{m^{(2)}}=\beta$. Тогда из $(24)$ и $(25)$ имеем

$$
\alpha^{2}+\beta^{2}=1, \quad \alpha \beta=0 .
$$

Отсюда следует, что

$$
\alpha=0,|\beta|=1 \quad \text { или } \quad|\alpha|=1, \beta=0 .
$$

Повторяя аналогично рассуждения для трех и более коэффициентов, отличных от нуля, мы неизменно приходим к тому, что лишь один коэффициент отличен от нуля и равен $\varepsilon= \pm 1$.

Итак,

$$
\overline{\mathscr{M}}_{N}(j)=\varepsilon j^{m}, \quad \text { где } \varepsilon= \pm 1, \quad|m|=k(n-2),
$$

что и требовалось доказать. 
4. Классификация алгебраической и неалгебраической интегрируемости динамических систем вида $(1),(2)$.

ОПРЕДЕЛЕНИЕ 1. Назовем минимальные однородные полиномы - решения системы уравнений (15) и (16), парииальными автоморфными формами для динамической системы $(1),(2)$ веса $m=\left(m_{1}, \ldots, m_{n}\right)$.

ОПРЕДЕЛЕНИЕ 2. Назовем динамическую систему (1), (2) в эквивалентной форме (12), (13) алгебраически интегрируемой, если она имеет $n-2$ независимых первых алгебраических интегралов $\psi_{i}(z), i=1,2, \ldots, n-2\left(\psi_{i}(\tilde{z})=\psi_{i}(z) \stackrel{\text { def }}{=} \gamma_{i}(w), \tilde{z}=z(w+1)\right)$, определяющих зависимость общего решения системы уравнений $(1),(2)$ от $n-2$ произвольных периодических функций $\gamma_{i}(w)$ периода 1 в дополнение к $\alpha(w)$ и $\beta(w)$ из $(5),(6)$.

Имеет место следующая теорема.

ТЕОРемА 2. Для того чтобы динамическая система (1), (2) была алгебраически интегрируема (в обычной терминологии - интегрируема), достаточно, чтобы она имела $2 n-2$ алгебраически независимые парииальные автоморфные формы $P_{k}^{i}$, $i=1,2, \ldots, 2 n-2$.

ДокАЗАТЕЛЬСтвО. Пусть мы имеем $2 n-2$ алгебраически независимые парциальные автоморфные формы $P_{k_{i}}^{(i)}, i=1,2, \ldots, 2 n-2$. Тогда образуем $n-2$ произведения $\psi_{i}, i=1,2, \ldots, n-2$,

$$
\psi_{i}(z)=\prod_{j=1}^{n}\left[P_{k_{j}}^{(j)}(z)\right]^{l_{j}}\left[P_{k_{n+i}}^{(n+i)}(z)\right]^{l_{n+i}}
$$

где $l_{j} \in \mathbb{Z}, j \in(1,2, \ldots, 2 n-2)$. При сдвиге $w \rightarrow w+1, z \rightarrow \tilde{z} \psi_{i}(\tilde{z})$ согласно (15), (11) примет вид

$$
\psi_{i}(\tilde{z})=\psi_{i}(z) \prod_{r=1}^{n} j_{r}{ }^{q_{r}(i)}
$$

где

$$
q_{r}^{(i)}=\sum_{s=1}^{n}\left(m_{r}^{(s)}-k_{s}\right) l_{s}+\left(m_{r}^{(n+i)}-k_{n+i}\right) l_{n+i} .
$$

Решая линейную систему из $n$ уравнений $q_{r}^{(i)}=0, r=1,2, \ldots, n$, относительно неизвестных $y_{j}=l_{j} / l_{n+i}, y_{j} \in \mathbb{Q}, j=1,2, \ldots, n$, мы получим $n-2$ набора решений для рациональных величин $y^{(i)}=\left(y_{1}^{(i)}, \ldots, y_{n}^{(i)}\right)$. Приведя каждьй из наборов $y^{(i)} \mathrm{k}$ общему знаменателю, мы получим $n-2$ набора целочисленных векторов $\left\{l_{j}^{(i)}, l_{n+i}^{(i)}\right\}$, $j=1,2, \ldots, n$, и, тем самым, $n-2$ первых интеграла (27).

Имеет место следующее утверждение, доказываемое аналогично.

Теорема 3. Если динамическая система (1), (2) имеет $2 n-2-l$ (әде $1 \leqslant l \leqslant n-2)$ алгебраически независимых автоморфных форм веса $m$, то она имеет $n-2-l$ nервых алгебраических интегралов. Если при әтом динамическая система (1), (2) 
имеет $l$ первых голоморфных неалгебраических интегралов, то такую систему будем называть неалгебраически интегрируемой.

Следует заметить, что если имеется хотя бы один первьй алгебраический интеграл, то такую систему также естественно назьвать алгебраически интегрируемой, поскольку она допускает понижение поря дка системы с помощью рациональной, эллиптической подстановки или подстановкой через тэта-функции рода $p \geqslant 2$, если род алгебраической кривой, задающей линии уровня первого интеграла, равен $p=0,1$ или $p \geqslant 2$ соответственно. Если после такой подстановки новая система не будет иметь ни одного алгебраического первого интеграла, а только необходимое число голоморфных первых интегралов, то такую систему следует называть неалгебраически интегрируемой. Разумеется, что это вопрос соглашения о терминологии.

Если динамическая система (1), (2) не является алгебраически интегрируемой, то вопрос существования голоморфных интегралов решается на основе локальной теории диффеоморфизмов.

Введем $x(w)=\left(x_{1}(w), \ldots, x_{n-1}(w)\right), x \in \mathbb{C}^{n-1}: z(w)=\left(x(w) z_{n}(w), z_{n}(w)\right)$. Тогда (см. (12)-(14))

$$
\begin{aligned}
x(-w) & =\bar{\Lambda}^{a} x(w), \\
x(w+1) & =\frac{\bar{\varphi}\left(x z_{n}, z_{n}\right)}{\varphi_{n}\left(x z_{n}, z_{n}\right)} \stackrel{\text { def }}{=} f(x), \\
z_{n}(w+1) z_{n}(w) & =\frac{\varphi_{n}(x, 1)}{\pi(x, 1)},
\end{aligned}
$$

где $\bar{\Lambda}_{i j}^{a}=\Lambda_{i j}^{a}, \bar{\varphi}_{k}=\varphi_{k}, f_{k}=\varphi_{k}(x, 1) / \varphi_{n}(x, 1), i, j, k=1, \ldots, n-1$.

Рассмотрим отображение (31) в окрестности неподвижной точки, которую без ограничения общности будем считать нулевой.

ОПРЕДЕЛЕНИЕ. Набор $\lambda=\left(\lambda_{1}, \lambda_{2}, \ldots, \lambda_{n}\right) \in \mathbb{C}^{n}$ собственных чисел линейной части отображения назьвается мультипликативно резонансным, если существует целочисленный вектор $m=\left(m_{1}, \ldots, m_{n}\right), m_{i} \geqslant 0,|m|=\sum m_{i} \geqslant 2$, такой, что для некоторого $j \in(1,2, \ldots, n)$ вьполняется равенство

$$
\lambda_{j}=\lambda^{m},
$$

где $\lambda^{m}=\lambda_{1}^{m_{1}} \cdots \lambda_{n}^{m_{n}}$.

В резонансном случае отображение приводится к нормальной форме на основе теоремы Пуанкаре-Дюлака. В [12], [32] дан пример неалгебраически интегрируемой динамической системы $(1),(2)$, сохраняющей площадь и имеющей резонанс. В нерезонансном случае имеет место следующая теорема Зигеля.

ОПРЕДЕЛЕНИЕ. Набор $\lambda \in \mathbb{C}^{n}$ назьвается набором мультипликативного типа $(C, \nu)$, если для всех $j \in(1,2, \ldots, n), m_{i} \in \mathbb{Z}_{+},|m|=\sum m_{i} \geqslant 2$, вьполняются неравенства

$$
\left|\lambda_{j}-\lambda^{m}\right| \geqslant C|m|^{-\nu}
$$


ТЕОРемА ЗИГЕЛЯ. Если набор собственных чисел линейной части голоморфного отображения в окрестности неподвижной точки имеет мультипликативный тип $(C, \nu)$ при некоторых $C>0, \nu>0$, то отображение биголоморфно әквивалентно в некоторой окрестности неподвижной точки своей линейной части (см. $[16$, с. 194$])$.

Таким образом, интегрирование отображения (31) зависит от принадлежности набора $\lambda$ мультипликативному $(C, \nu)$ типу.

Следующая важная теорема из [16] утверждает, что почти все наборы собственных значений вектора $\lambda=\left\{\lambda_{i}\right\} \in \mathbb{C}^{n}$ являются наборами мультипликативного $(C, \nu)$ типа и мы можем использовать теорему Зигеля.

Теорема [16, теорема 4, с. 172]. Мера множества точек, не являющихся ни при каком $C>0$ точками $(C, \nu)$ типа, равна нулю, если $\nu>(n-2) / 2$.

Но эта теорема не дает ответа на вопрос: является ли данный набор собственных чисел мультипликативного $(C, \nu)$ типа или нет.

Оказьвается, что имеет место следуюшая теорема [34], [33], дополняющая теорему Зигеля и теорему, приведенную вьше.

Теорема 4 [34], [33] (следствие теорем Бэйкера-Фельдмана). Набор $\lambda \in \mathbb{C}^{n}$ uмеет мультипликативный тип $(C, \nu)$, если набор $\lambda$ мультипликативно нерезонансен $и$ числа $\lambda_{i}$ принадлежат $\mathbb{A}$ - алгебрачческому числовому полю.

Следует сделать следующее замечание. После выдающихся работ А. О. Гельфонда и Т. Шнайдера по решению 7-й проблемы Гильберта А. Бэйкер развивает новые мощные методы в теории трансцендентных чисел [17], [35], [18], [36] в 1964-1970 гг. (см. лекцию А. Бэйкера как филдсовского медалиста [35]), на основе которых он доказывает [17] впервые очень важную теорему об оценке линейных форм логарифмов алгебраических чисел относительно специальной характеристики алгебраического числа - его высоты. Н. И. Фельдман доказьвает после А. Бэйкера аналогичную теорему [19], но относительно длины алгебраического числа (см. определение высоты и длины алгебраического числа, например, в [20, Дополнение Д, с. 267-271], см. также [37]). Хотя именно эта теорема оказывается более подходящей для нас для прямого доказательства [34], [33] диофантовых условий в теореме Зигеля, мы предлагаем впредь, в отличие от [34], [33], цитировать обе теоремы под общим названием теоремы Бэйкера-Фельдмана. Фактически, такая справедливая оценка этих результатов, важных для использования в теории динамических систем, содержится в [34], [33]. А. Бэйкер неоднократно улучшал оценки линейных форм логарифмов алгебраических чисел, доведя ее совместно с Г. Вюстхольцем до трудно превьшаемого предела в [38].

В работах [34], [39] на основе теоремы 4 и теоремы Зигеля проинтегрированы динамические системы $(1),(2)$ с матрицей кроссинг-симметрии $A^{\text {Chew-Low }} A(l, 1)$, совпадающей при $l=1$ с матрицей из работ [12], [32]. Заметим, что при исследовании динамики квадратичных отображений в [40] Ю. Мозером были также использованы результаты теорем Бэйкера-Фельдманапри доказательстве вьполнимости некоторого диофантового условия в теореме Рюссманна.

5. Дополнение и заключение. В работе [41], предварительная версия которой докладьвалась на Международном математическом конгрессе в 1998 г. в Берлине, на 
международных конференциях, посвященных 90-летию Л. С. Понтрягину и Д. И. Блохинцева, а также на VI конференции по разностным уравнениям в Познани [42], была развита общая теория интегрирования функционально-разностньх автономных уравнений для двух неизвестных комплексных функций $z(w): \mathbb{C} \rightarrow \mathbb{C}^{2}$ от коплексной переменной переменной $w$ следующего вида $(n=2)$ :

$$
z(\psi(w))=F(z(w)), \quad z \in \mathbb{C}^{n}, \quad w \in \mathbb{C}, \quad \psi \in \operatorname{Aut}(\mathbb{C})
$$

Здесь $\psi, F$ суть произвольные заданные отображения, $F: z \mapsto z^{\prime}=F(z)$ - бирациональное отображение из группы $\operatorname{Bir} C^{n}$ всех автоморфизмов $\mathbb{C}^{n} \rightarrow \mathbb{C}^{n}$ с коэффициентами из некоторого алгебраически замкнутого поля $K$ (при частном выборе $\psi(w)=w+1$ система (34) имеет привычный вид функционально-разностных уравнений в комплексной области).

Алгебраическая интегрируемость уравнений (34) определена в [41] через существование инвариантного линейного пространства-линейной системы алгебраических кривых степени $\mu$, рода $p \geqslant 0$ и размерности $k \geqslant 1$ (см. более детально [41]). Решение этой проблемы при $n=2$ в [41] делает актуальньм исследование возможностей обобщения алгебро-геометрического подхода работы [41] на случай $n \geqslant 3$. Поскольку при $n \geqslant 3$ имеются трудные, пока нерешенные проблемы в самой алгебраической геометрии, то является актуальным исследование проблемы интегрирования более простых систем уравнений $(1),(2)$, или в эквивалентной форме $(12),(13)$, для накопления опыта и обобщения подхода [41] для $n \geqslant 3$. Это особенно важно в виду возможных приложений этого подхода к интегрированию обкновенных и частных дифференциальных уравнений (см. [41]).

Автор глубоко благодарен В. И. Арнольду, В. В. Козлову и В. Б. Приезжеву за неоднократные полезные обсуждения и поддержку. Особо признателен автор А. Бэйкеру и Н. И. Фельдману за важные для теоремы 4 обсуждения оценок линейных форм логарифмов алгебраических чисел. Автор также благодарен за полезные обсуждения С. В. Болотину, А. Д. Брюно, С. А. Довбьшу, И.М. Кричеверу, Н. Г. Мощевитину, Ю. В. Нестеренко и М.Б. Севрюку. И, наконец, автору приятно поблагодарить А. Бэйкера и Р. С. Маккэя за гостеприимство во время пребьвания в Кэмбриджском университете и Тринити-колледже.

\section{СПИСОК ЦИТИРОВАННОЙ ЛИТЕРАТУРЫ}

[1] Moser J. On the theory of quasi-periodic motions // SIAM Rev. 1966. V. 8. P. 145-172.

[2] Moser J. K. Convergent series expansions for quasi-periodic motions // Math. Ann. 1967. V. 169. P. 136-176.

[3] Moser J. K. Stable and Random Motion in Dynamical Systems. Princeton: Princeton Univ. Press, 1973.

[4] Arnol'd V.I. Reversible systems // Nonlinear and Turbulent Processes in Physics / ed. R.Z. Sagdeev. Harwood: Chur, 1984. P. 1161-1174.

[5] Arnol'd V. I., Sevryuk M. B. Oscillations and bifurcations in reversible systems // Nonlinear Phenomena in Plasma Physics and Hydrodynamics / ed. R. Z. Sagdeev. Moscow: Mir, 1986. P. 31-64.

[6] Sevryuk M. B. Reversible Systems. Lecture Notes in Math. V. 1211. Berlin: Springer, 1986. 
[7] Devaney R. L. Reversible diffeomorphisms and flows // Trans. Amer. Math. Soc. 1976. V. 218. P. 89-113.

[8] Quispel G.R.W., Roberts J. A.G., Thompson C. J. Integrable mappings and soliton equations // Phys. D. 1989. V. 34. P. 183-192.

[9] Hudson H. Cremona Transformations in Plane and Space. Cambridge: University Press, 1927.

[10] Математическая энциклопедия. Т. 3. М.: Советская энциклопедия, 1982.

[11] Ширков Д. В., Серебряков В. В., Мещеряков В. А. Дисперсионные теории сильных взаимодействий при низких энергиях. М.: Наука, 1967.

[12] Rerikh K. V. Cremona transformation and general solution of one dynamical system of the static model // Phys. D. 1992. V. 57. P. 337-354.

[13] Moser J. On the integrability of area-preserving Cremona mappings near an elliptic fixed point // Bol. Soc. Mat. Mexicana. 1960. P. 176-180.

[14] Веселов А. П. Группа Кремоны и динамические системы // Матем. заметки. 1989. Т. 45. C. $118-120$.

[15] Арнольд В.И., Ильяшенко Ю.С. Обькновенные дифференциальные уравнения // Итоги науки и техники. Совр. пробл. матем. Фундамент. направления. Т. 1. М.: ВИНИТИ, 1985. C. $7-149$.

[16] Арнольд В. И. Дополнительные главы теории обыкновенных дифференциальных уравнений. М.: Наука, 1978.

[17] Baker A. Linear forms in logarithms of algebraic numbers // Mathematika. 1966. V. 13. P. 204-216.

[18] Baker A. Transcendental Number Theory. Cambridge: Cambridge University Press, 1990.

[19] Feldman N. I. Improvement of evaluation of the linear form of logarithms of algebraic numbers // Mat. Sbornik. 1968. V. 77 (119). № 3. P. 423-436.

[20] Фельдман Н.И. Седьмая проблема Гильберта. М.: Изд-во МГУ, 1982.

[21] Гантмахер Ф.Р. Теория матриц. М.: Наука, 1966.

[22] Мещеряков В. А. Метод построения некоторых классов решений уравнений типа Чу-Лоу // Препринт ОИЯИ Р-2369. Дубна: ОИЯИ, 1965.

[23] Fairlie D. B. Structure of the crossing matrix for arbitrary internal symmetry groups // J. Math. Phys. 1966. V. 7. P. 811-815.

[24] Wanders G. Analytic structure of the scattering matrix in the neutral pseudoscalar theory // Nuovo Cimento. 1962. V. 23. P. 817-837.

[25] Мешеряков В. А. Метод решения уравнений типа уравнений Чу-Лоу // Препринт ОИЯИ Р-1965. Дубна: ОИЯИ, 1965..

[26] Rothelutner T. Exacte Lösungen von Low-Geichungen // Z. Phys. 1964. V. 177. P. 287-299.

[27] Журавлев В. И., Мешеряков В. А., Рерих К.В. Об одном новом виде решений уравнений типа уравнений Чу-Лоу // ЯФ. 1969. Т. 10. С. 168-175.

[28] Meshcheryakov V.A., Rerikh K. V. A method of the local construction of invariant subspaces in the space of solutions of the Chew-Low type equations // Ann. Phys. 1970. V. 59. P. 408-425.

[29] Гердт В. П., Мещеряков В. А. Локальная форма решения уравнений Чу-Лоу // ТМФ. 1975. T. 24. C. $155-163$.

[30] Рерих К. В. Уравнения Чу-Лоу как преобразования Кремона. Структура общих интегралов // ТМФ. 1982. Т. 50. С. 251-260.

[31] Rerikh K. V. Chew-Low equations as the Cremona transformation. Structure of the first integral and general solution // Proc. of the XIII Int. Conf. on Differential Geometric Methods in Theoretical Physics (Shumen, Bulgaria, 1984). Singapore: World Scientific, 1986. P. 170-178.

[32] Rerikh K. V. Non-algebraic integrability of one reversible Cremona dynamical system. The Poincaré (1.1) resonance and the Birkhoff-Moser analytical invariants // Proc. of Internat. Workshop "Finite-Dimensional Integrable Systems". Dubna: JINR, 1995. P. 171-180.

[33] Rerikh K. V. Algebraic addition concerning the Siegel theorem on the linearization of a holomorphic mapping // Math. Z. 1997. V. 224. P. 445-448. 
[34] Rerikh K. V. Non-algebraic integrability of the Chew-Low reversible dynamical system of the Cremona type and the relation with the 7 th Hilbert problem (non-resonant case) // Phys. D. 1995. V. 82. P. 60-78.

[35] Baker A. Effective methods in the theory of numbers // Proceedings of the International Congress of Mathematicians (Nice, September 1970). V. 1. Paris: Gauthier-Villars, 1971. P. 19-26.

[36] Baker A. // Selected Studies: Physics-Astrophysics, Mathematics, Hystory of Science / ed. T.M. Rassias, G. M. Rassias. A Volume Dedicated to the Memory of Albert Einstein (Scientific Research and Technology Agency, Athens, Greece). Amsterdam-New York-Oxford: North-Holland, 1982.

[37] Гекке Э. Лекции по теории алгебраических чисел. М., 1940.

[38] Baker A., Wüstholz G. Logarithmic forms and group varieties // J. Reine Angew. Math. 1993. V. 442. P. 19-62.

[39] Rerikh K. V. Non-algebraic integrability of one reversible dynamical system of the Cremona type // J. Math. Phys. 1998. V. 39. P. 2821-2832.

[40] Moser J. On quadratic symplectic mappings // Math. Z. 1994. V. 216. P. 417-430.

[41] Rerikh K. V. Integrability of functional equations defined by byrational mappings (general theory of integration of birational cascades and cascade-flows). II // Math. Physics, Analysis and Geometry (to appear).

[42] Rerikh K. V. Integrability of functional equations defined by plane byrational mappings // The Electronic Data Base of Abstracts. Section "Dynamical Systems and Differential Equations" of International Congress of Mathematicians (August, 18-27, Berlin, 1998); // Abstracts "Optimal Control" and Appendices of International Conference Dedicated to the 90th Anniversary of L. S. Pontryagin (August, 31-September, 6, Moscow, 1998). P. 298. 